the line of sight, and suitably mounted and clock driven. Such an instrument has been constructed and is in operation at the Ohio State University.

A photocell at the focus communicates the changing brightness pattern on the rectangular objective to a recorder or counter. As the mirror is rotated, the orientation that gives the minimum count is found to correspond, as previously announced, to the direction of winds aloft, and the count value to their velocity.

The low cost and rapid method described offers a possibility of widespread measures of directional stellar scintillation for meteorological, observatory site testing, or other purposes.

This work has been supported in part by contract with the Geophysics Research Directorate, Air Force Cambridge Research Center.

McMillin Observatory, Ohio State University, Columbus, Ohio.

\section{Huang, Su-Shu. Problems of accretion and star formation.}

The effect of a magnetic field, of stellar and interstellar origin, on the rate of accretion is investigated. It is found that with a field strength of $10^{-6}$ gauss in interstellar space, accretion becomes negligible. Therefore very luminous stars do not come into being through rejuvenation of less luminous stars.

We propose that stars are formed in the compressed layer when two clouds collide. It thereby provides a mechanism for the formation of the early-type stars the existence of which Oort (1954) has assumed in his theory of formation of $\mathrm{O}$ associations. We emphasize a two-dimensional gravitational collapse in the compressed layer which removes two difficulties usually encountered in condensation theories of star formation, i.e., (I) excessive mass and (2) excessive angular momentum of the condensation.

A rough calculation of the frequency of formation of binary stars in the proto-star stage is also undertaken. According to a previous paper (Huang and Struve 1954), we have suggested that the angular momenta of rotating stars in a cluster, or an association, are derived from collisions in their proto-star stages. The frequency of collisions is proportional to the velocity dispersion in the cluster. On the other hand, the frequency of formation of binary stars according to our calculation is inversely proportional to the fifth power of the velocity dispersion. It follows therefore that in a cluster where one finds larger than average rotation, there should be fewer binary stars. In the Pleiades where most earlytype stars are rotating rapidly, Struve (private communication) found few spectroscopic binary stars in a systematic search for such objects.

Huang, S. S. and Struve, O. 1954, Ann. Astroph. 17, 85.

Oort, J. H. 1954, B. A. N. 12, 177 .

$$
\begin{array}{r}
\text { Leuschner Observatory, } \\
\text { University of California, } \\
\text { Berkeley, Calif. }
\end{array}
$$

\section{Hynek, J. A. and Stanger, P. C. The composite-} spectrum star 5 Lacertae.

The variability of the composite-spectrum star 5 Lacertae in radial velocity and in spectrum, announced by one of the authors in 1938, has been studied in greater detail with aid of plates taken at the Perkins Observatory.

It is found that (a) the incomplete velocity curve of the primary, ascribable to orbital motion, indicates a period in excess of 50 years and an orbit of high eccentricity (b) sharp $\mathrm{H}$ and $\mathrm{K}$ lines, ordinarily associated with the secondary star in spectroscopic binaries of this type, yield a velocity curve largely in phase with the primary curve but displaced to negative values; they are variable in intensity and show emission edges, and the sharp $\mathrm{K}$ line is not always "filled in" in the manner characteristic of $\mathrm{K}$ lines in composite spectra of this type; these features suggest an expanding shell about the primary star which, at certain phases, also envelops the secondary, and (c) during the next few years this pair can be expected to have a separation about $\mathrm{O}$. I, placing it at the limit of resolution of large instruments.



Johnson, Hugh M. Is the Galaxy a barred spiral?

If the Galaxy is actually a barred spiral, should we certainly know it now? The question is not so radical if one thinks of short-barred spirals such as NGC 1398. This note suggests that some features of a barred spiral may be observed in the Galaxy. Let us first consider the inclination of the spiral arms to the radius vector. It is about $65^{\circ}$ according to the Yerkes optical observations, but is nearly $90^{\circ}$ according to the Leiden $2 \mathrm{I}-\mathrm{cm}$ observations. The discrepancy may be at least partly removed by various dynamical hypotheses 